\title{
On the Underlying Cognitive Adaptations for Extended Phenotype Expression and Evaluation
}

\author{
Tran Dinh $^{1} \cdot$ Steven W. Gangestad ${ }^{1}$
}

Received: 16 January 2019 / Revised: 17 January 2019 / Accepted: 24 January 2019 /

Published online: 2 February 2019

(C) Springer Nature Switzerland AG 2019

Luoto (2019) puts forward a bold, updated view of human sexual selection. In this view, perspectives on human sexual selection to date have not fully recognized the importance of extended phenotypes - non-bodily environmental manifestations of behavior that affect the fitness of individuals, such as housing, creative outputs, and displays of status or wealth. To the extent that these outputs function as signals of qualities valued in mates, they may importantly affect mate choice and, thereby, sexual selection.

Dawkins (1982) developed the concept of extended phenotypes because traditional views of phenotypes did not fully account for how genes affect inclusive fitness. The concept of extended phenotypes elevates the profile of behavior in understanding important outcomes of selection, as behavior often affects fitness by manipulating the environment the organism lives within. Humans specialize in manipulating environments in fitness-enhancing ways. Extraction of high-quality energy sources (highlighted as a driver of an evolved human adaptive complex in some formulations; e.g., Kaplan et al. 2000) typically involves environmental manipulation. Human intellectual capacities - e.g., abilities to model cause and effect sequences that permit novel solutions to adaptive problems (Tooby and DeVore 1987) - arguably evolved partly or even primarily through effects on extended phenotypes. Though these capacities perhaps initially evolved primarily through effects on nutrient capture, they drive technological innovation and development more broadly.

\section{Theoretical Challenges}

There is little doubt, then, that extended phenotypes have been important to humans' abilities to populate virtually every habitat found on earth. But Luoto (2019) particularly emphasizes the implications of these observations for an understanding of human sexual selection. Human extended phenotypes can either directly offer fitness benefits

Tran Dinh

trandinh@unm.edu

1 Department of Psychology, University of New Mexico, Albuquerque, NM, USA 
to mates or indirectly relate to future fitness benefits to mates via underlying qualities they tap. Hence, mate choosers may benefit by attending to extended phenotypes. In turn, to the extent that individuals reap benefits in the form of higher quality or greater numbers of mating opportunities via their production of extended phenotypes, sexual selection may (a) boost selection for production of these extended phenotypes, above and beyond that selected through other effects on fitness, and (b) select for means of displaying extended phenotypes to potential mates. Extended phenotypes may hence come to possess signaling qualities.

We agree with this analysis as far as it goes. In our view, however, it does not go far enough. It does not specify or delineate the psychological nature of receiver adaptations that lead them to value extended phenotypes. And it does not adequately specify the psychological nature of signaler adaptations that lead them to display extended phenotypes.

Take, for instance, three instances of extended phenotypes that Luoto (2019) argues may be favored by mates: a nice apartment; a luxury car; an artifact of creative production. Luoto recognizes that these extended phenotypes may benefit mates for different reasons. A nice apartment directly affects offspring fitness. A luxury car may be associated with resources or status more generally. Creative production may be associated with innovativeness, which may directly benefit offspring through its outcomes or indirectly benefit offspring through genetic effects (e.g., Miller 2000). But what, then, is the nature of the adaptations through which these features affect mate choice? One might imagine that individuals have the capacity to "compute" the fitness value of each extended phenotype via effects on one's own reproduction. But how is that fitness value arrived at, especially within a contemporary setting in which luxury cars and exclusive apartments are novelties?

In our view, evolved mate evaluation adaptations that take into account extended phenotypic productions must be capable of extracting information that is represented at a level that would have been recurrent during the period in which the adaptations evolved. What we see lacking in the target article is a proposal of how nice apartments, luxury cars, and creative products yield information that is represented at a level recurrent in human ancestry, upon which preferences are based. At the very least, just as different adaptations purportedly underlie evaluation of distinct physical features (even if individual evaluations are integrated at some level of overall mate evaluation), different adaptations would likely be responsible for evaluation of different extended phenotypes. But, should they exist, what are such adaptations? Individuals can likely appreciate the innovativeness of creative adaptive solutions and thereby evaluate underlying features (e.g., intelligence) responsible for them. But such evaluations do not speak to the evolved processes through which nice apartments and luxury cars affect mate choice. Absent a fuller explication of the architectural nature of adaptations that respond to extended phenotypes, the fuller sexual selection theory that Luoto invites us to consider remains an as-yet unfulfilled promissory note.

Using reasoning from life history theory on trade-offs in energy allocation decisions, Luoto (2019) also hypothesizes that individuals with fast life history strategies trade off investment in extended phenotype expression for accelerated reproduction. Here, too, we think additional theory is needed. Kaplan et al. (2000, 2003) proposed that individual investments in embodied capital in the form of large brains and the time and energy dedicated to developing its capacities (e.g., learning) co-evolved with long lifespans, across which those investments can pay off. These investments may also have evolved to be 
conditional, such that the extended phenotypes resulting from human innovation are produced more under conditions that favor longevity. At the same time, certain forms of extended phenotypes - particularly ones with sexually selected benefits - may especially benefit males seeking fitness returns via higher mating and reproductive success under conditions less propitious to long lives. It is therefore not clear that (or when) "fast" versus "slow" strategies should reliably predict sexually selected extended phenotype expression; it may depend on as-yet-unspecified particulars.

\section{Empirical Evaluation}

In an empirical test of his proposals, Luoto (2019) finds that the Economic Complexity Index (ECI) of nations can be predicted from environmental features such as atmospheric cold demands and pathogen prevalence. Economic complexity, he argues, is partly and importantly an outcome of sexually selected signaling via innovative production of extended phenotypes. Furthermore, individuals are expected to invest most in such innovative production where slow life histories are favored, e.g., where cold demands are great. For several reasons, we are concerned that the regression models conducted may not accurately reflect the true differential impacts of environmental features on outcomes.

(a) High levels of multicollinearity may introduce instability to parameter estimates. Luoto chose to omit pathogen prevalence due to collinearity with cold demands, based on small differences in model fit with one or the other included. On a priori bases, however, pathogen prevalence may plausibly serve as an ancestrallyrecurrent cue of relatively extrinsic mortality risk. Fit could be affected by differential validity of environmental indices; cold demands are likely measured with better validity than pathogen prevalence.

(b) A causal model derived from the stated hypotheses could not be adequately tested from the data available or statistical tests performed. For one, spurious model results can arise from simultaneous entry of a mixture of "extrinsic" causes (e.g., cold demands, pathogen prevalence) and their proposed life history outcomes (e.g., adolescent fertility rates, intelligence) as predictors of another life history outcome (i.e., ECI) in a multiple regression. It is not clear why other co-evolved life history outcomes should be controlled.

Furthermore, correlations between variables are not sufficient evidence to suggest evolved processes affecting trade-offs between early reproduction and extended phenotype expression. Correlations may well be affected by modern evolutionary novelties that need not reflect evolved processes. Comparative data reveal that hunter-gatherer women have higher fertility than nonhuman primates, despite slower human life histories of prolonged juvenile periods and delayed sexual maturity for development of embodied capital (Kaplan et al. 2000). Yet in contemporary developed nations, girls reach menarche earlier but tend to have lower fertility as adults. This is likely due to high energy availability and accessibility of modern forms of contraception. How do evolutionary novelties figure into evolved processes regulating fertility outcomes? The cognitive 
mechanisms that assess modern contraception may not be the ones evolved for fertility regulation.

(c) Finally, data are nested and require modeling (e.g., mixed model analysis) to account for nesting. Nations within the same geographical region are expected to cluster on predictor and outcome variable values. Data were collected in 2016 and 1995 "to control for the effects of time," yet the effects of time were not modeled.

These methodological concerns can potentially be addressed; perhaps results are robust despite them. Aside from these particulars, however, it is not clear to us why sexually selected signaling is required to explain the pattern of effects. Once again, independent of its signaling properties, investment in innovativeness - and the "embodied capital" that facilitates it (Kaplan et al. 2000, 2003) — may be more strongly favored where extrinsic mortality rates are lower (e.g., in relative absence of pathogens). While the ideas and hypotheses Luoto (2019) puts forward are interesting and thought-provoking, the data he presents do not adequately test ideas specific to his proposed updated sexual selection theory.

\section{Compliance with Ethical Standards}

Conflicts of Interest On behalf of all authors, the corresponding author states that there is no conflict of interest.

Publisher's Note Springer Nature remains neutral with regard to jurisdictional claims in published maps and institutional affiliations.

\section{References}

Dawkins, R. (1982). The extended phenotype. Oxford UK: Oxford University Press.

Kaplan, H. S., Hill, K., Lancaster, J. B., \& Hurtado, A. M. (2000). A theory of human life history evolution: Diet, intelligence, and longevity. Evolutionary Anthropology, 9, 156-185.

Kaplan, H., Lancaster, J., \& Robson, A. J. (2003). Embodied capital and the evolutionary economics of the human life span. Population and Development Review, 29, 152-182.

Luoto, S. (2019). An updated theoretical framework for human sexual selection: From ecology, genetics, and life history to extended phenotypes. Adaptive Human Behavior and Physiology.

Miller, G. F. (2000). The mating mind. New York: Doubleday.

Tooby, J., \& DeVore, I. (1987). The reconstruction of hominid behavioral evolution through strategic modeling. In W. Kinzey (Ed.), Primate models of hominid behavior (pp. 183-2371). New York: State University of New York Press. 